\title{
Giant Magnetostriction Jumps in Conventional NbTi Superconductor
}

A. NabiąeK ${ }^{a, *}$, S. VAsiliev ${ }^{a, b}$, V. Chabanenko ${ }^{b}$, P. Aleksyeyev ${ }^{a, b}$, V. Rusakov ${ }^{c}$, S. Piechota $^{a}$

AND H. SZYMCZAK ${ }^{a}$

${ }^{a}$ Institute of Physics, Polish Academy of Sciences

al. Lotników 32/46, 02-668 Warsaw, Poland

${ }^{b}$ Donetsk Physico-Technical Institute, Ukrainian Academy of Sciences

72 R. Luxemburg str., 83114 Donetsk, Ukraine

${ }^{c}$ Donetsk National University, 24 Universitetskaya str., 83-055 Donetsk, Ukraine

Magnetostriction of a large slab of conventional NbTi superconductor, with a diameter of about $6 \mathrm{~mm}$, was investigated by the strain gauge technique. At the temperature of $4.2 \mathrm{~K}$ and in the external magnetic field of several $\mathrm{T}$ we found irreversible magnetostriction of an order of $10^{-5}$, where the number means the relative changes of the slab diameter. This large magnetostriction is accompanied by giant magnetostriction jumps, caused by thermomagnetic avalanches. These thermomagnetic avalanches also cause giant jumps of the surface magnetization, which can be observed by using the Hall probes. The results were analyzed in the framework of the pinning induced magnetostriction model.

PACS numbers: 74.25.Qt, 74.70.Ad, 75.80.+q

\section{Introduction}

Magnetostriction of several conventional superconductors was, for the first time, investigated by Brändli in late sixties of the twentieth century [1]. However, the investigated conventional superconductors were characterized by high purity and also by low critical current density. The experimentally observed magnetostriction was relatively low, of an order of $10^{-7}$, and no magnetostriction jumps were observed. Pinning induced magnetostriction model was, for the first time, proposed by Ikuta et al. to explain giant magnetostriction of a large single crystal of high temperature $\mathrm{Bi}_{2} \mathrm{Sr}_{2} \mathrm{CaCu}_{2} \mathrm{O}_{8}$ superconductor [2]. According to this model

*corresponding author; e-mail: nabia@ifpan.edu.pl 
the magnetostriction of the superconductor depends on both the critical current density and the sample dimensions. Pinning induced magnetostriction is large in large superconducting samples characterized by strong critical current densities.

Large superconducting samples characterized by strong critical current densities are also susceptible to the occurrence of thermomagnetic instabilities called flux jumps. Flux jumps are problematic from the viewpoint of practical applications of the superconductors, because they may drive the superconducting sample into a normal or, at least, into the resistive state [3]. By decreasing sample diameter it is possible to avoid flux jumping. Hence, commercially available superconducting leads are made as composites, consisting of a large number of thin superconducting wires surrounded by a matrix, made from a normal metal. Such composites are not susceptible to the flux jumps and one may also expect their magnetostriction to be very small.

According to the pinning induced magnetostriction model flux jumps induce giant jumps of magnetostriction. Giant magnetostriction jumps were, for the first time, discovered in large crystals of high temperature superconductors $[4,5]$. Recently, we have discovered giant magnetostriction jumps in conventional $\mathrm{Nb}_{3} \mathrm{Al}$ superconductor [6], which, in comparison to other conventional superconductors, is characterized by relatively strong critical current density and large second critical field, $H_{\mathrm{c} 2}$. In our present work we investigate magnetostriction and magnetostriction jumps in a large slab of conventional NbTi superconductor.

\section{Experimental technique}

Our NbTi-50\% superconductor was obtained during a technological process, described in Refs. [7, 8]. This technological process leads to a large amount of non-superconducting inclusions of $\alpha$-Ti phase, which act as pinning centers. From the cylinder obtained after the extrusion process, we cut a parallelepiped with dimensions $6 \times 10 \times 10 \mathrm{~mm}^{3}$. During our experiments the external magnetic field was parallel to one of the $10 \mathrm{~mm}$ edges of the investigated sample. The external magnetic field was also parallel to the axis of the original cylinder.

In our magnetostriction experiments we measured the relative changes of the slab diameter in the external magnetic field. We applied the strain gauge technique. One of the strain gauges was glued onto the surface of the investigated sample. The second strain gauge was glued onto the surface of a reference sample. The reference sample was made from quartz. These two strain gauges were connected to the two arms of AC resistance bridge.

Surface magnetization measurements were done by using two Hall probes. The Hall probes were made from tin doped InSb films [8]. One of the Hall probes was placed in the center of the $6 \times 10 \mathrm{~mm}^{2}$ face of our parallelepiped. This face was perpendicular to the external magnetic field. This Hall probe measured the local surface induction, $B_{\text {surf }}$, at the surface of our slab. The second Hall probe measured the induction of the external magnetic field, $B_{\text {ext }}=\mu_{0} H_{\text {ext }}$. The 
difference between the signals from our two Hall probes was proportional to the local surface magnetization, $M_{\text {surf }}=B_{\text {surf }}-B_{\text {ext }}$.

We performed our experiments in superconducting magnet with the maximal attainable field of $12 \mathrm{~T}$. The magnetic field was swept with the rate of $1 \mathrm{~T} / \mathrm{min}$. We measured magnetostriction and local surface magnetization at the temperature of $4.2 \mathrm{~K}$. During measurements, the investigated sample was immersed in liquid helium.

\section{Experimental results}

Figure 1 presents a hysteresis loop of the transverse magnetostriction of our NbTi slab, taken at $4.2 \mathrm{~K}$. The maximal external magnetic field applied in this experiment was about $12 \mathrm{~T}$. As one can see in Fig. 1, in the external magnetic field of several $\mathrm{T}$ we have found large, of an order of $10^{-5}$, irreversible magnetostriction. In the external magnetic field higher than the irreversibility field, $H_{\mathrm{irr}}$, the observed magnetostriction curve becomes reversible. In our case $H_{\mathrm{irr}} \approx 11 \mathrm{~T}$. The behavior of the magnetostriction hysteresis loops of type II superconductor is very similar to that of the well known hysteresis loops of magnetization and can be well understood in the framework of the pinning induced magnetostriction model [2]. In Fig. 1 one can also see giant magnetostriction jumps. Most of the magnetostriction jumps occur in the external magnetic field lower than about $3 \mathrm{~T}$.

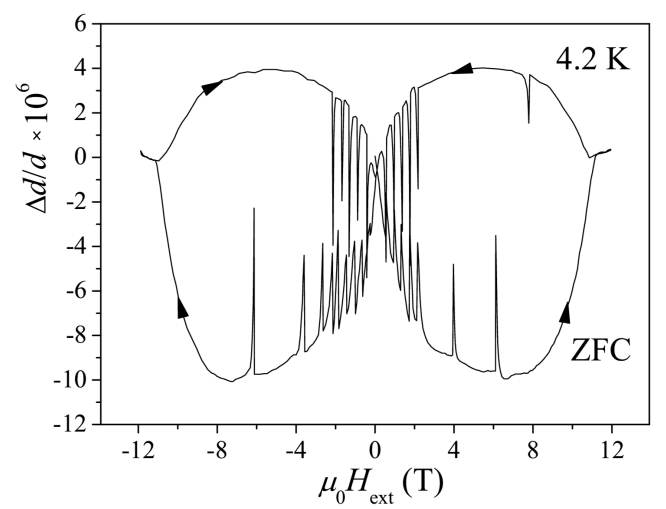

Fig. 1. Transverse magnetostriction hysteresis loop of the $6 \times 10 \times 10 \mathrm{~mm}^{3} \mathrm{NbTi}-50 \%$ slab, taken at $4.2 \mathrm{~K}$ with the external magnetic field parallel to the slab surface. After cooling in zero external magnetic field, the external magnetic field was first increased to $12 \mathrm{~T}$ (the ZFC curve), then decreased to $-12 \mathrm{~T}$ and, at the end, increased to zero.

In order to study the structure of the magnetostriction jumps in more details, we have also registered the magnetostriction hysteresis loop with the maximal applied external magnetic field of about $2.8 \mathrm{~T}$, and this loop is shown in Fig. 2. In Fig. 2 it is clearly seen that during each jump the diameter of the investigated slab changes rapidly, approaching the value, which the sample has after cooling in zero 
external magnetic field. This diameter corresponds to $\Delta d / d=0$ in Fig. 2. After a sudden magnetostriction jump the dimensions of the sample change relatively slowly. While the external magnetic field is changed, after the jump, by about $0.3 \mathrm{~T}$ the magnetostriction, $\Delta d / d$, approaches to the value which was observed before the jump. The structure of the observed magnetostriction jumps is similar to the jumps of the magnetization, caused by the thermomagnetic avalanches [3].

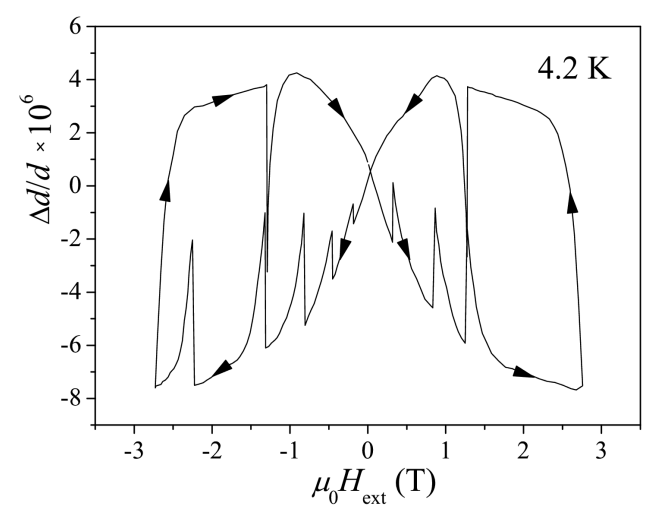

Fig. 2. Transverse magnetostriction hysteresis loop of the $6 \times 10 \times 10 \mathrm{~mm}^{3} \mathrm{NbTi}-50 \%$ slab, taken at $4.2 \mathrm{~K}$ with the external magnetic field parallel to the slab surface. The external magnetic field was first increased to $2.8 \mathrm{~T}$, then decreased to $-2.8 \mathrm{~T}$ and, at the end, increased to zero.

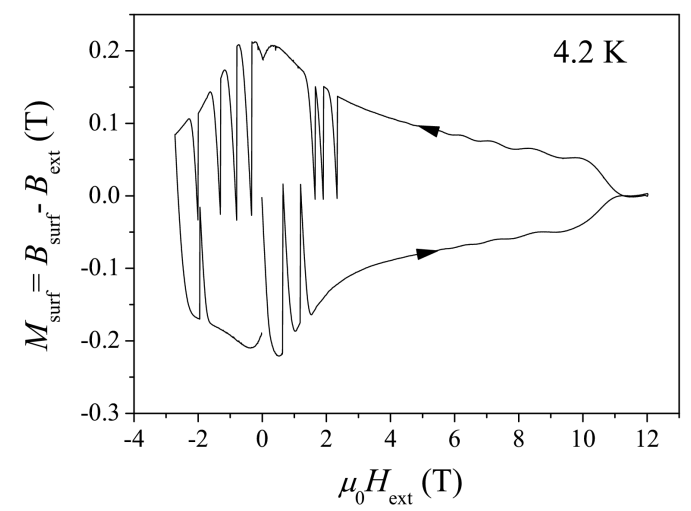

Fig. 3. The hysteresis loop of the surface magnetization, $M_{\text {surf }}$, of the $6 \times 10 \times 10 \mathrm{~mm}^{3}$ NbTi- $50 \%$ slab, taken at $4.2 \mathrm{~K}$. The magnetization was measured by using Hall probes, one of which was placed in the middle of the $6 \times 10 \mathrm{~mm}^{2}$ face, perpendicular to the external magnetic field.

Figure 3 shows the hysteresis loop of the surface magnetization of the investigated NbTi slab, taken at the same conditions as the magnetostriction hysteresis 
loop, shown in Fig. 1. In Fig. 3 one can see giant jumps of surface magnetization. The jumps of the surface magnetization occur in the same range of the external magnetic field as the jumps of the magnetostriction. The irreversibility field of the loop shown in Fig. 3 is the same as that of the loop shown in Fig. 1.

In the external magnetic field higher than about $5 \mathrm{~T}$ one can see in Fig. 3 that the measured $M_{\text {surf }}$ signal begins to oscillate in external magnetic field. The amplitude of these oscillations increases with increasing external magnetic field. However, the observed oscillations are not connected with the properties of the investigated NbTi sample, but with the properties of the applied Hall probes. This phenomenon is caused by the Shubnikov-de Haas oscillations of the Hall voltage. In the case of the applied Hall probes and in the investigated range of temperatures and magnetic fields, the Shubnikov-de Haas oscillations are usually smaller than $0.25 \%$ of the total Hall voltage [9]. However, the full compensation of these oscillations was in our experiment impossible and they are visible as tiny waves of the $M_{\text {surf }}\left(H_{\text {ext }}\right)$ signal. This signal was several orders of magnitude lower than the signals taken from each of the applied Hall probes, separately.

\section{Analysis and discussion}

We used the results of our surface magnetization measurements, presented in Fig. 3, for the estimations of the critical current density as well as for the simulations of the magnetostriction curve in frames of the pinning induced magnetostriction model. The estimation of the critical current density is usually done on the basis of magnetic moment measurements. However, large superconducting samples, like our NbTi slab, cannot be put into commercially available magnetometers. Hence, for the estimations of the critical current density another techniques must be used.

According to the critical state model [10], critical current density is proportional to the slope of the magnetic field profile in the investigated sample. In the case of an infinite slab in the external magnetic field parallel to its surface the critical current density, $j_{\mathrm{c}}$, is given by the equation: $j_{\mathrm{c}}(x)=-\frac{1}{\mu_{0}} \frac{\partial B(x)}{\partial x}$, where $x$ is the coordinate perpendicular to the slab surface. Neglecting demagnetizing effects and assuming the superconducting sample to be fully penetrated by the magnetic flux and the density of the screening current, equal to $j_{\mathrm{c}}$, to be constant over the whole sample volume, we may calculate $\left|j_{\mathrm{c}}\right|=M_{\text {surf }} / \mu_{0} d$, where $M_{\text {surf }}$ is the surface magnetization measured in our experiment in the center of a slab with diameter $2 d$. At the temperature of $4.2 \mathrm{~K}$ and in zero external magnetic field, this estimation gives the critical current density of $5.3 \times 10^{3} \mathrm{~A} / \mathrm{cm}^{2}$.

The relative changes of an infinite slab diameter, $\Delta d / d$, caused by the pinning forces can be calculated according to formula [2]:

$$
\frac{\Delta d}{d}=-\frac{1}{2 E \mu_{0} d} \int_{0}^{d}\left[B_{\text {ext }}^{2}-B^{2}(x)\right] \mathrm{d} x
$$

where $E$ is the Young modulus and $B_{\text {ext }}=\mu_{0} H_{\text {ext }}$ is the induction of the external 


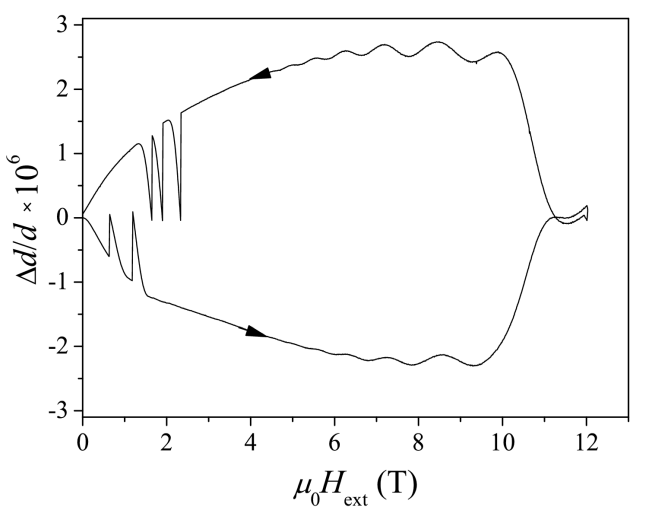

Fig. 4. Transverse magnetostriction hysteresis loop calculated in the framework of the pinning induced magnetostriction model by using the surface magnetization data, presented in Fig. 3. The Young modulus was assumed to be $E=80 \mathrm{GPa}$.

magnetic field. The integration in Eq. (1) can be easily done, if we assume the slope of the magnetic field profile, $\partial B(x) / \partial x$, to be constant over the whole sample volume. If we make the same assumptions as in the above described estimation of the critical current density, we can transform Eq. (1) into the following one:

$$
\frac{\Delta d}{d}=\frac{1}{2 E \mu_{0}} M_{\text {surf }}\left(B_{\text {ext }}+\frac{1}{3} M_{\text {surf }}\right) .
$$

We used this simple equation for the simulation of the magnetostriction curve presented in Fig. 3. In our simulations we used the Young modulus $E=$ $80 \mathrm{GPa}$ [11]. The width of the simulated hysteresis loop of the magnetostriction (Fig. 4) is smaller, however, of the same order of magnitude as the experimental one (Fig. 1). The visible in the strong magnetic field oscillations of the simulated magnetostriction curve result from the Shubnikov-de Haas oscillations of the Hall voltage, as we discussed earlier. The structure of the simulated magnetostriction jumps is similar to the experimental ones (see Fig. 1 and Fig. 2).

\section{Conclusions}

Giant magnetostriction and magnetostriction jumps also occur in conventional NbTi superconductor. These phenomena occur if the superconducting sample is large and, additionally, characterized by strong critical current density. Surface magnetization measurements can be directly used in order to simulate magnetostriction curves. This method of simulations is particularly useful in the case of large superconducting samples, whose magnetic properties cannot be studied by other experimental techniques.

\section{Acknowledgments}

This work was partly supported by the State Committee for Scientific Research (Poland) under contract No. 4 T10B 02325. 


\section{References}

[1] G. Brändli, Phys. Kondens. Mater. 11, 111 (1970).

[2] H. Ikuta, N. Hirota, Y. Nakayama, K. Kishio, K. Kitazawa, Phys. Rev. Lett. 70, 2166 (1993).

[3] R.G. Mints, A.L. Rakhmanov, Rev. Mod. Phys. 53, 551 (1981).

[4] V.V. Chabanenko, I.B. Krynetskii, S. Piechota, H. Szymczak, Physica B 216, 289 (1996).

[5] A. Nabialek, P. Komorowski, M.U. Gutowska, M.A. Balbashov, J.N. Górecka, H. Szymczak, O.A. Mironov, Supercond. Sci. Technol. 10, 786 (1997).

[6] A. Nabiałek, V.V. Chabanenko, V.F. Rusakov, S. Vasiliev, N.N. Kabdin, H. Szymczak, S. Piechota, B.N. Kodess, S.A. Kononogov, J. Low Temp. Phys. 139, 239 (2005).

[7] O.V. Chernyi, N.F. Andrievskaya, V.O. Ilicheva, G.E. Storozhilov, P.J. Lee, A.A. Squitieri, Adv. Cryogen. Eng. 48, 883 (2002).

[8] L. Cooley, P. Lee, D. Larbalestier, in: Handbook on Superconducting Materials, Vol. 1, Superconductivity, Materials, and Processes, Eds. D.A. Cardwell, D.S. Ginley, Institute of Physics Publishing Ltd., Bristol 2003, p. 603; http://www.asc.wisc.edu/pdf_papers/630.pdf.

[9] O.A. Mironov, M. Myronov, S. Durov, O. Drachenko, J. Leotin, Physica B 346347, 548 (2004).

[10] C.P. Bean, Phys. Rev. Lett. 8, 250 (1962).

[11] E.W. Collins, Applied Superconductivity, Metallurgy and Physics of Titanium Alloys, Plenum Press, New York 1986. 